\title{
Further Study on Gardiac Hypertrophy in Spontaneously Hypertensive Rats
}

\author{
Kikuko ImaMura* Hitoshi TANiJIRI, ${ }^{*}$ Masakazu Ireda, * \\ Tadasu TAkatsu, ${ }^{*}$ Chujiro KashiI, ${ }^{* *}$ and Keishiro Kawamura***
}

There are numerous investigations concerning cardiac hypertrophy in all aspects. In previous studies we investigated on cardiac hypertrophy in SHR histochemically and morphologically. The present experiments dealt with detailed studies on the ultrastructural alterations suggesting sarcomerogenesis and stereological observations. Furthermore, we examined the relationship between the cardiocytes and capillaries, which are of particular significance in the vital functions of the myocardium, and directed the attention to the characteristic behavior of the capillaries in cardiac hypertrophy of SHR.

\section{Materials and Methods:}

SHR $\left(F_{19-26}\right)$ and control animals of Wistar-Kyoto were used. The systolic blood pressure and body weight were measured once a week. For the histological study, animals were sacrificed at $3,5,9,11,15$, and 21 weeks and 1 year of life. The specimens were taken from the posterior papillary muscle and the lateral wall of the left ventricle. One $\mathrm{mm}$ cubes from each specimen were fixed with glutaraldehyde-osmium acid and embedded in epon. Semithin sections of $1 \mu$ were cut, stained with toluidine blue and observed by light microscopy. These sections were also used for the measurement of the cardiocyte diameter as well as length, and the counting of the number of capillaries and cardiocytes. Subsequently ultrathin sections were cut and examined by electron microscopy. Stereology was made by the method of Weibel.

\section{Results:}

1) Blood pressure: In SHR it elevated gradually from the 7 th week after birth and was over $180 \mathrm{mmHg}$ after the 15th week. On the other hand, it was always lower than $140 \mathrm{mmHg}$ in control rats. 2) Heart weight/body weight ratio: It was largest, approximately $5.5 \times 10^{-3}$ at the 3 rd week in both groups and thereafter rapidly fell until the 13 th week to be $3.5 \times 10^{-3}$ and $3.8 \times 10^{-3}$, in SHR and control rats, respectively, After the 15 th week it

From the Third Division, Department of Internal Medicine, Osaka Medical College*, Takatsuki, Osaka; Department of Internal Medicine, Osaka Red Cross Hospital, Osaka**; and Third Division, Department of Internal Medicine, Faculty of Medicine, Kyoto University***, Kyoto. 
continued to increase to $4.8 \times 10^{-3}$ at 1 year in SHR, while it remained constant in controls, 3) Cardiocyte diameter: In the posterior papillary muscle of the left ventricle of SHR it was significantly greater from the 5 th week than in controls, it reached $22 \mu$ at the 11th week and was almost the same throughout the experimental period. On the other hand, the cardiocyte diameters of the left ventricular lateral wall in SHR showed a significant difference after the 11 th week as compared with controls, where it was $20 \mu$ and $18 \mu$ in SHR and controls, respectively. Thereafter it continued to increase gradually in SHR, while in controls it remained $18 \mu$ in both areas of the left ventricle. 4) Cardiocyte length: In the posterior papillary muscle of the left ventricle it increased to over $100 \mu$ from the 11th week in SHR. On the other hand, it ranged between $80 \mu$ and $100 \mu$ in controls. 5) Histological findings: The connective tissue proliferation was observed in all over the myocardium after the 15th week of SHR, where the medial hyperplasia as well as the intimal thickening appeared in some arteries and artcrioles. Electronmicroscopically sarcomerogenesis was observed in the myocardium of SHR from the 15th week, which was characterized by an appearance of $Z$-substance in sarcomere and subsarcolemmal dense mats accompanied by myofilamentous structures. Moreover, distortion of the intercalated discs became remarkable with an increase in foldings. 6) Stereologic measurement: In SHR the myofibrillar fractional volume increased and the mitochondrial fractional volume decreased significantly at the 15 th and 21 st week in the posterior papillary muscle of the left ventricle. As a result, the myofibrils/mitochondria ratio increased significantly. At the same time the fractional volume of $\mathrm{T}$-system increased significantly. 7) Capillary/cardiocyte ratio: At the 3rd week there were more cardiocytes than capillaries in the posterior papillary muscle of the left ventricle in both groups. At the 5 th week it became $1: 1$ in both groups. While this relationship remained in the control rats throughout the period of study, it increased significantly in SHR from the 9th to 21st week. 8) Capillary concentration (number of capillaries $/ \mathrm{mm}^{2}$ ): At the $3 \mathrm{rd}$ week it was largest and rapidly reduced to $2300 / \mathrm{mm}^{2}$ at the 9 th week in the posterior papillary muscle of the left ventricle in both groups. And then it was almost the same in controls, whereas in SHR it increased at the 11th and the 15th week. 9) "Tunnel" capillary: A capillary, which passes through a tunnel made of the sarcolemma in the center of the cardiocyte, was observed in SHR after the 11 th week. Serial transverse sections revealed that a capillary in the deep cleavage of the cardiocyte soon located in the "tunnel "within the cardiocyte and then was finally found in the divided cardiocyte.

In cardiac hypertrophy of SHR the diameter as well as the length of cardiocytes increased. Ultrastructurally, sarcomerogenesis was found out in the 
myocardium. The quantitative analysis by stereology revealed that the myofibrils/mitochondria ratio increased significantly reflecting not only an increase in the fractional volume of myofibrils but also a decrease in the mitochondrial volume. The additional data regarding the capillary density indicated an increase in the capillary/cardiocyte ratio. Moreover, the appearance of "tunnel" capillaries seen in hypertrophied myocardium might be considered to be a representation of the adaptation in cardiac hypertrophy. 